\title{
High-Resolution CT Chest Findings in Suspected COVID-19 Pneumonia Patients With Negative Real-Time Polymerase Chain Reaction Assay
}

\author{
Shazia Yusuf ${ }^{1}$, Hafsah Ahmad ${ }^{1}$, Romasa Zeb ${ }^{1}$, Uswa Zeb ${ }^{1}$, Ahmed A. Zeb ${ }^{1}$ \\ 1. Diagnostic Radiology, Capital Hospital, Islamabad, PAK
}

Corresponding author: Hafsah Ahmad, drhahmadkharal@gmail.com

\section{Abstract \\ Objective}

The study was conducted with the objective of describing High-resolution computed tomography (HRCT) chest findings of clinically suspected COVID-19 patients having a negative real-time polymerase chain reaction (RT-PCR) assay as well as prevalence and distribution of the HRCT chest manifestations consistent with the diagnosis of COVID-19 pneumonia.

\section{Methods}

This descriptive cross-sectional study was conducted prospectively on a total of 48 patients with high clinical suspicion for COVID-19 and a negative RT-PCR assay that was presented to the Diagnostic Radiology Department of Capital Hospital, Islamabad from July 2020 to December 2020. These patients were included via non-probability consecutive sampling, had a positive history of contact with a known COVID-19 patient and/or any two of the following signs and symptoms; fever, cough, malaise, body aches, arthralgia, newonset loss of taste and smell, and dyspnea or oxygen saturation less than $85 \%$. A detailed history was sought after informed consent and all these patients underwent non-contrast HRCT chest scans that were reported by an experienced consultant radiologist. The scans showing positive features for COVID-19 pneumonia were assessed for the nature and distribution of the disease.

\section{Results}

Amongst 48 suspects with negative RT-PCR assay, 38 (79.2\%) showed ground-glass opacities, a hallmark feature of COVID-19 pneumonia. A total of 22 (57.89\%) of these 38 patients had ground-glass opacities with a crazy-paving pattern, nine (23.68\%) mixed ground-glass opacities with consolidation, and seven (18.42\%) had pure ground-glass opacities. Among these $79.2 \%$ suspects, ground-glass opacities were multifocal in 37 (97.37\%), bilateral in 35 (92.10\%), peripheral in 36 (94.74\%), and dorsally located in $32(81.6 \%)$ cases. Subpleural atelectatic bands were seen in 18 (47.36\%) of these, bronchovascular markings were prominent in 15 (39.47\%), and reverse halo sign was positive in nine (23.68\%) cases. Out of the rest of the cases, three were diagnosed as interstitial lung disease, two as chronic lung disease, and one as active pulmonary tuberculosis.

Review began 02/27/2021 Review ended 03/16/2021 Published 03/21/2021

\section{(c) Copyright 2021}

Yusuf et al. This is an open access article distributed under the terms of the Creative Commons Attribution License CC-BY 4.0., which permits unrestricted use, distribution, and reproduction in any medium, provided the original author and source are credited.

\section{Conclusion}

The majority of clinically suspected cases for COVID-19 showed hallmark findings on non-contrast HRCT chest scans in keeping with coronavirus disease regardless of a negative RT-PCR assay.

Categories: Radiology

Keywords: crazy paving, covid-19, hrct chest, rt-pcr, ground glass opacities, subpleural atelectatic bands, reverse halo sign

\section{Introduction}

COVID-19 caused by SARS-CoV-2 is a highly infective disease that has caused a pandemic infecting more than 103 million people in over 200 countries with deaths over two million. More than 75 million people have recovered from the disease worldwide [1,2]. There is no certainty about the spread, signs/symptoms, diagnosis, and clinical management of coronavirus disease so far [3]. Minor symptoms include a slight fever, mild cough, malaise, body aches, arthralgia, myalgia, and new-onset loss of taste and/or smell. Patients with moderate disease present with signs and symptoms of pneumonia being severe cough, dyspnea, and respiratory distress that progress to sepsis, respiratory and multi-organ failure in the grave stage [4,5]. All the patients showing any of the earlier described signs and symptoms with a travel history to a country with the rapid spread of COVID-19 or a contact history with a known COVID-19 patient within the last 14 days are considered highly suspicious [6]. 
The clinical manifestations of COVID-19 along with the positive nasopharyngeal and/or oropharyngeal realtime reverse transcription polymerase chain reaction (RT-PCR) assay are considered the standard of reference for diagnosing the disease these days [7]. The sensitivity of the RT-PCR assay is approximated to around $60-71 \%$ [8]. A high number of false negatives are likely because of ongoing genomic mutations of SARS-CoV-2, technical and operator ee, lack of resources, and prowess needed for this genomic test $[6,9]$.

A high-resolution computed tomography (HRCT) chest is a rapid diagnostic technique for COVID-19 that has an added benefit of determining the severity, complications, and treatment plan for infected patients [10]. HRCT chest findings typical of COVID-19 pneumonia include bilateral multifocal peripheral-based ground-glass opacities [11].

HRCT has a higher sensitivity of approximately $89 \%$ and a moderate specificity of up to $68 \%$ [12]. Many types of research show HRCT to be one step ahead of RT-PCR as a first-line screening investigation for COVID-19 in emergency and hospital settings owing to its higher sensitivity and propose the idea of using chest CT or HRCT as a standard of reference along with clinical and laboratory evaluation for the diagnosis of COVID-19 $[9,13]$.

In this study, we determine the HRCT findings in patients clinically suspected of COVID-19 pneumonia with a negative RT-PCR assay. We also aim to assess the nature and distribution of HRCT lesions suggestive of COVID-19 pneumonia in these suspected cases.

\section{Materials And Methods}

This prospective, cross-sectional descriptive study was conducted at the Imaging unit of the Diagnostic Radiology Department, Capital Hospital, Capital Development Authority (CDA), Islamabad for a duration of six months, from July 2020 to December 2020.

After receiving an approval letter from the ethical committee of the hospital, informed consent and detailed history of signs and symptoms were sought from a total of 48 COVID-19 suspected patients with negative RT-PCR tests. The subjects were selected via non-probability consecutive sampling. These were entitled patients and thus underwent HRCT chest scans free of cost. Some of these patients had a positive history of contact with a known COVID-19 patient and some presented with any two of the following signs/symptoms; fever, cough, malaise, body aches, arthralgia, new-onset loss of taste and smell, dyspnea, or oxygen saturation less than $85 \%$. All known cases of asthma, tuberculosis, lung carcinoma, and lung metastases were excluded from the study. Children less than 14 years, pregnant and lactating mothers were not included in the study on account of high radiation dose.

A total of 48 patients were scanned in a 64-Slice Toshiba Aquilion CT Scanner (Canon Medical Systems Corporation, Otawara-shi, Tochigi, Japan). Patients were positioned supine with feet first and were scanned in a craniocaudal direction from the apex of lungs to diaphragm without any intravenous contrast agent. Scans were performed in full inspiration by instructing patients to hold their breath for seven to eight seconds. The technical parameters were kept at $120 \mathrm{kVp}, 300 \mathrm{mAs}$, and a rotation time of $0.5 \mathrm{sec}$. Scanning slice thickness was $2 \mathrm{~mm}$ with an intersection interval of $10 \mathrm{~mm}$ that was reduced to $0.5 \mathrm{~mm}$ after automatic reconstruction. The scanner was properly disinfected after each suspected COVID-19 patient was scanned.

The reconstructed HRCT images were transferred to Vitrea software at the workstation. Coronal, sagittal, and axial views were assessed on lung and mediastinal windows. Initially, the scan was analyzed by a senior postgraduate resident with the scan being distributed into two categories; suggestive and non-suggestive of COVID-19 pneumonia and was reported within the first 24 hours of scanning by a senior consultant radiologist having 19 years of experience. Evaluation of the scan was based on the type, size, and distribution of opacities with associated bronchovascular and fibrotic changes. The ground-glass opacities were classified as with or without consolidations on the basis of the obscuration of vessels. The ground-glass opacities were further divided into with or without a crazy-paving pattern on thickening of intralobular or interlobular septae. The distribution was explained as peripheral or central based on the involvement of outer one-third and inner two-third of lung parenchyma respectively and ventral or dorsal via a presumed central line. The lung parenchymal lesions were also described in terms of laterality and lobar involvement. The associated findings that strengthened the diagnosis of COVID-19 were also noted, these included subpleural atelectatic bands, prominent bronchovascular markings, and reverse halo signs.

Data entry and analysis were done by using IBM SPSS Statistics 23 and HRCT chest findings for suspected patients with distribution and nature of lesions were expressed as frequencies and percentages.

\section{Results}

Out of a total of 48 patients with clinical suspicion of COVID-19 pneumonia and negative RT-PCR swab, $62.5 \%(n=30)$ were males, $37.5 \%(n=18)$ were females. The mean age of the study group was 48.4 years (range $=30-90$ years). The median interval between the RT-PCR test and HRCT scan was found to be one day (range $=0-3$ days). $97.9 \%$ of the total suspected patients presented with fever and body aches, $68.7 \%$ with arthralgia or myalgia, $52 \%$ with cough, $33.3 \%$ with new-onset loss of taste and smell, $20.8 \%$ with shortness 


\section{Cureus}

of breath, and oxygen saturation less than $85 \%$ in room air.

Among the total patients, 79.2\% $(\mathrm{n}=38)$ had HRCT findings typical of COVID-19, $12.5 \%(\mathrm{n}=6)$ had findings suggestive of other diagnoses and 8.3\% $(n=4)$ had normal HRCT chest scans (Figure 1). The patients with other HRCT findings were diagnosed with different lung disorders (Table 1).

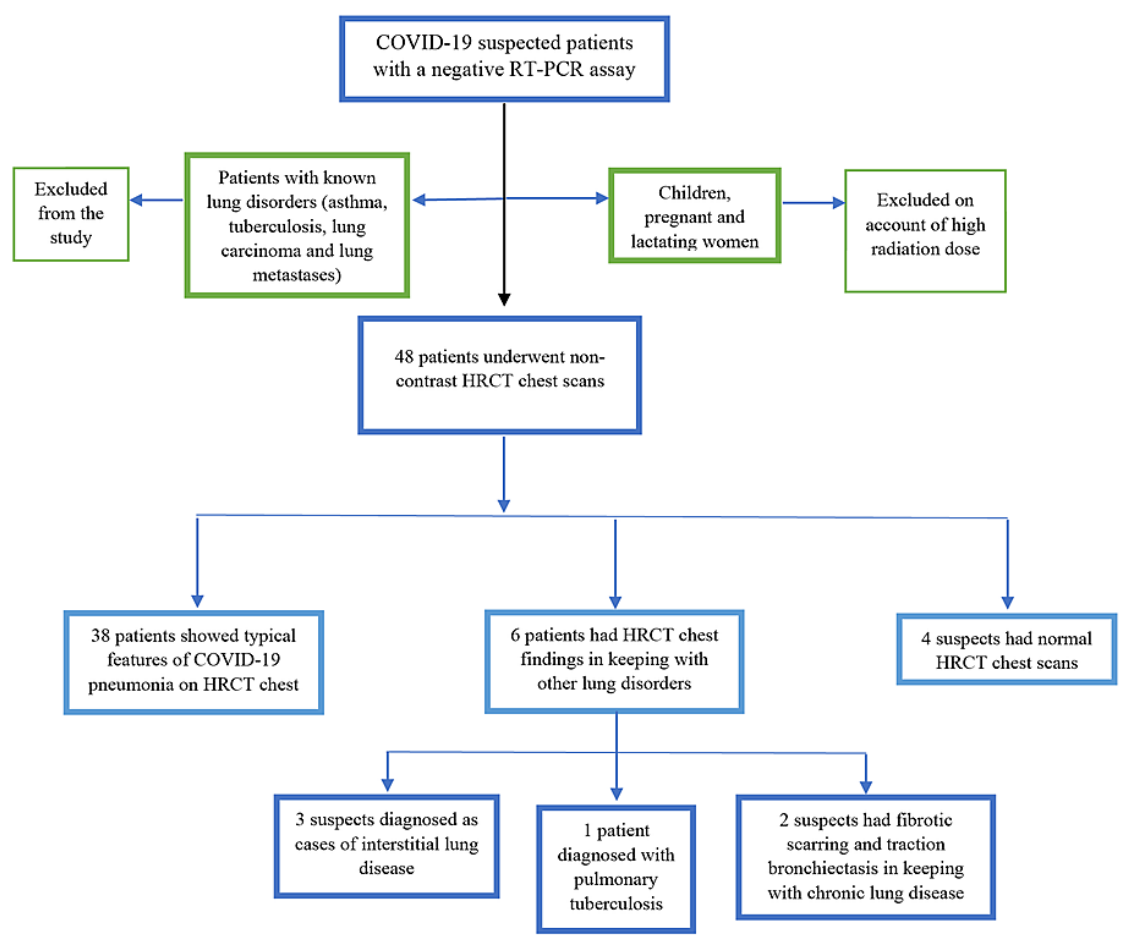

FIGURE 1: Study flowchart.

COVID-19: coronavirus disease 2019; HRCT: High-resolution computed tomography; RT-PCR: Real-time polymerase chain reaction. 


\section{Cureus}

\begin{tabular}{|c|c|c|c|}
\hline S. No & Radiological findings & Number of patients & Diagnosis \\
\hline 1 & Honeycombing/interstitial thickening & 3 & Interstitial lung disease \\
\hline 2 & Fibrotic scarring & 2 & Chronic lung disease \\
\hline 3 & Bronchiectasis & 2 & Chronic lung disease \\
\hline 4 & Emphysematous lungs with cysts and bullae & 2 & Chronic lung disease \\
\hline 5 & Pleural effusions & 1 & Tuberculosis \\
\hline 6 & Nodules & 1 & Tuberculosis \\
\hline 7 & Cavitary lesions & 1 & Tuberculosis \\
\hline 8 & Lymphadenopathy & 1 & Tuberculosis \\
\hline
\end{tabular}

TABLE 1: HRCT chest findings suggestive of other diagnoses.

HRCT: High-resolution computed tomography.

Hallmark radiological finding suggestive of COVID-19 pneumonia - ground glass opacification (GGO) was found in 38 patients. These were further categorized as pure GGO, GGO mixed with consolidation, and GGO with a crazy-paving pattern. $57.89 \%$ of the patients showed GGO mixed with crazy paving pattern whereas GGO with consolidation and pure GGO were found in $23.68 \%$ and $18.42 \%$ of the cases, respectively (Figures 2, 3, 4, 5, 6; Table 2). The additional findings that supported the diagnosis of COVID-19 were subpleural atelectatic bands, prominent bronchovascular markings, and reverse halo signs. Subpleural atelectatic bands were found in 18 patients and bronchovascular markings were prominent in 15 patients (Figure 4; Table 2). A reverse halo sign was seen in only nine of the 38 patients (Table 2). 


\section{Cureus}

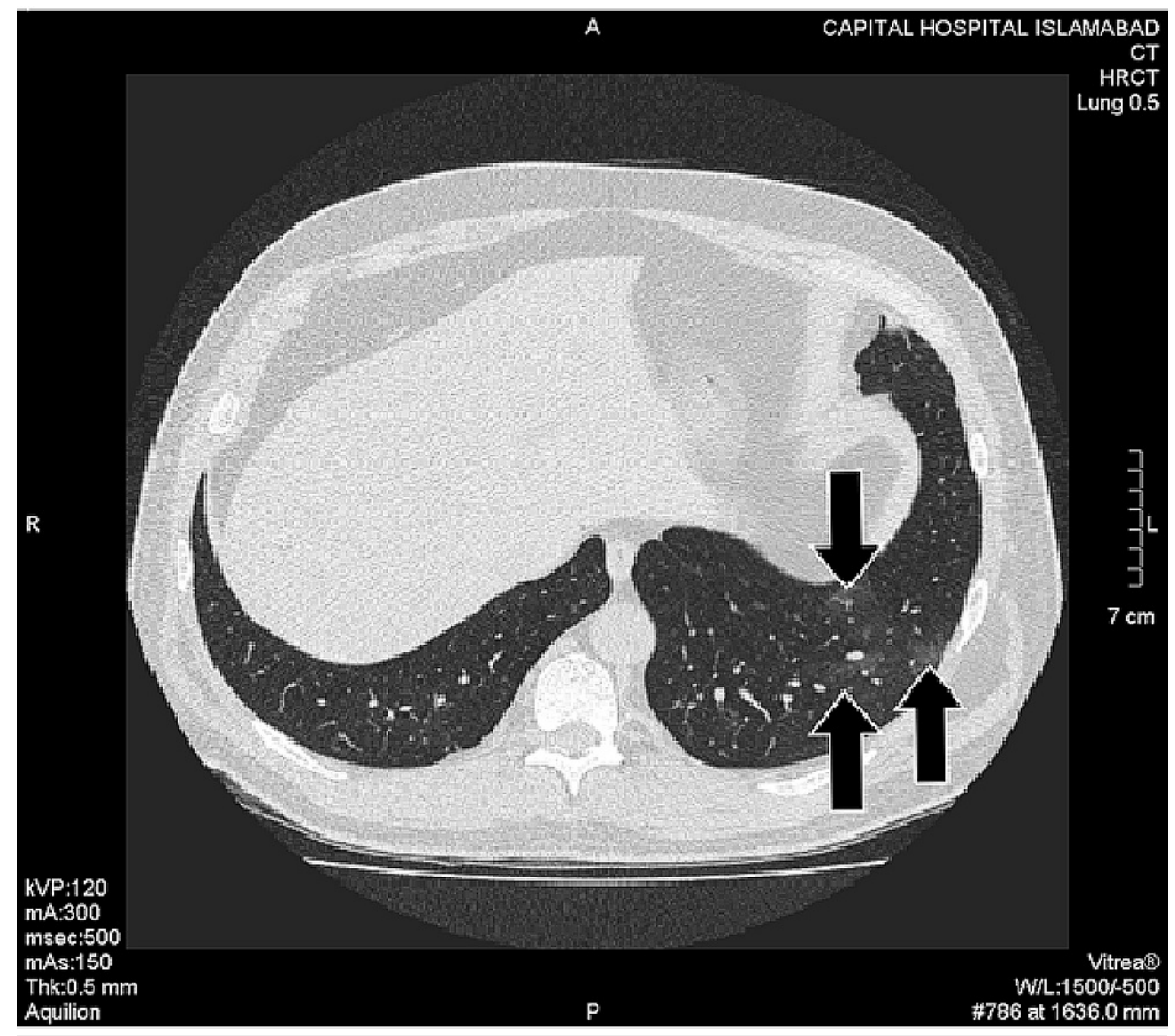

FIGURE 2: A COVID-19 RT-PCR negative patient with fever and newonset loss of taste and smell.

The axial slice of HRCT scan in lung window shows small patches of pure ground-glass opacities scattered in the peripheral and dorsal distribution in the left lower lobe (black arrows).

HRCT: High-resolution computed tomography; RT-PCR: Real-time polymerase chain reaction. 


\section{Cureus}

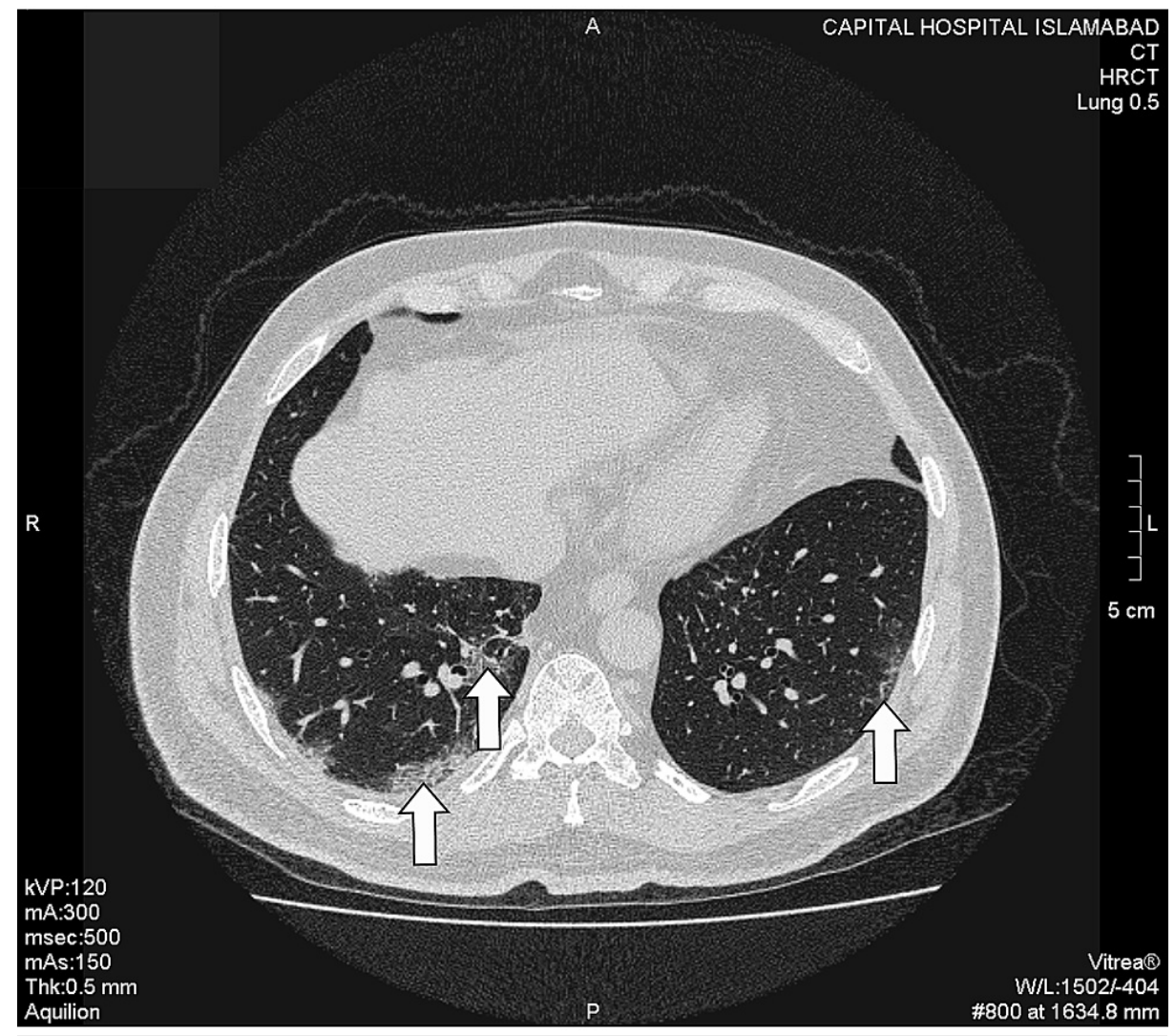

FIGURE 3: A male patient with cough and shortness of breath.

HRCT demonstrates few peripheral and dorsal ground-glass opacities with a crazy-paving pattern in bilateral lower lobes (white arrows).

HRCT: High-resolution computed tomography. 


\section{Cureus}

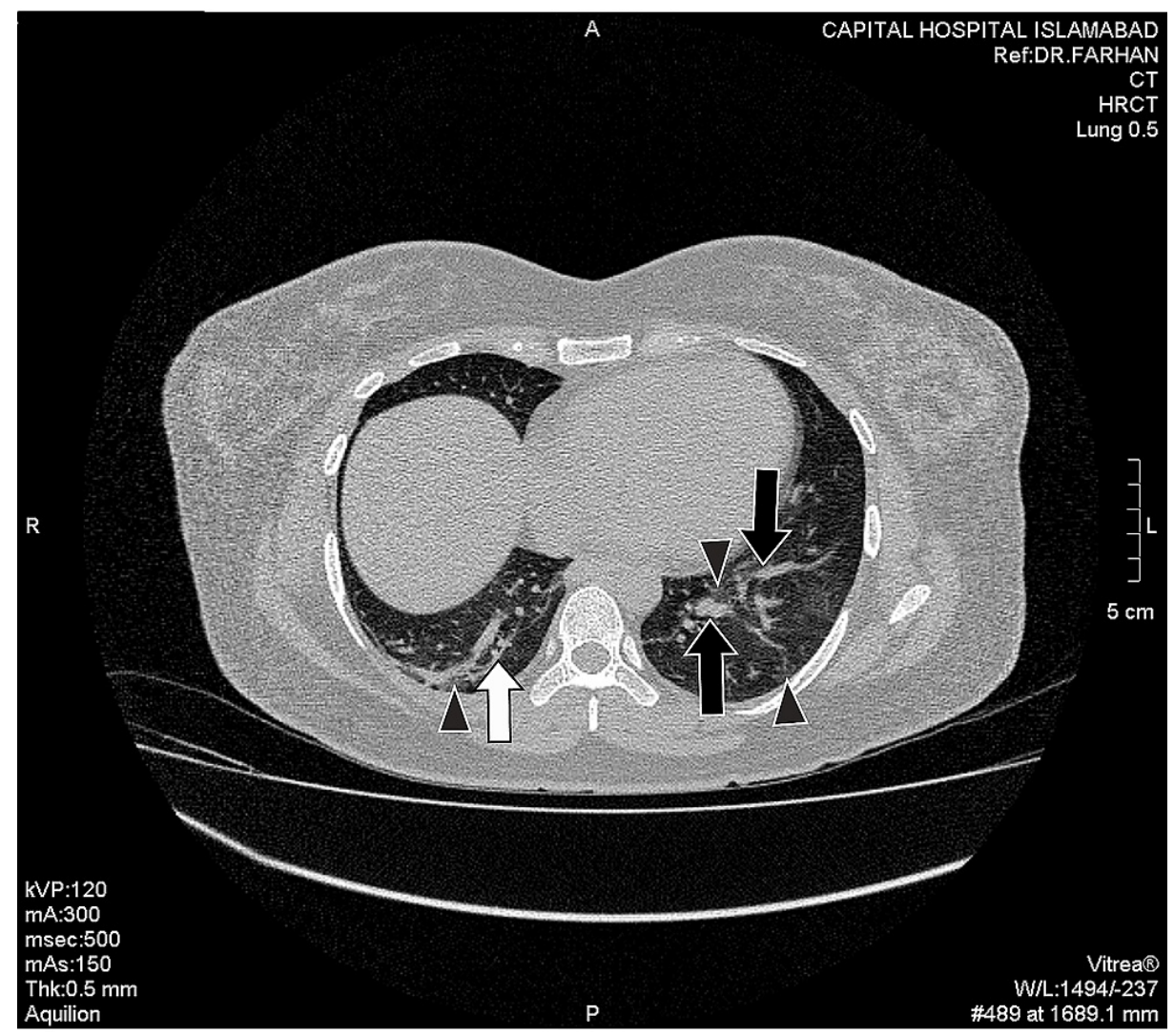

FIGURE 4: A middle-aged female presented with cough, fever, and body aches on the fourth day of symptoms.

HRCT scan illustrates bilateral areas of ground-glass opacities (black arrowheads), the subpleural atelectatic band in right lower lobe (white arrow), and prominent bronchovascular markings in the left lower lobe (black arrows).

HRCT: High-resolution computed tomography. 


\section{Cureus}

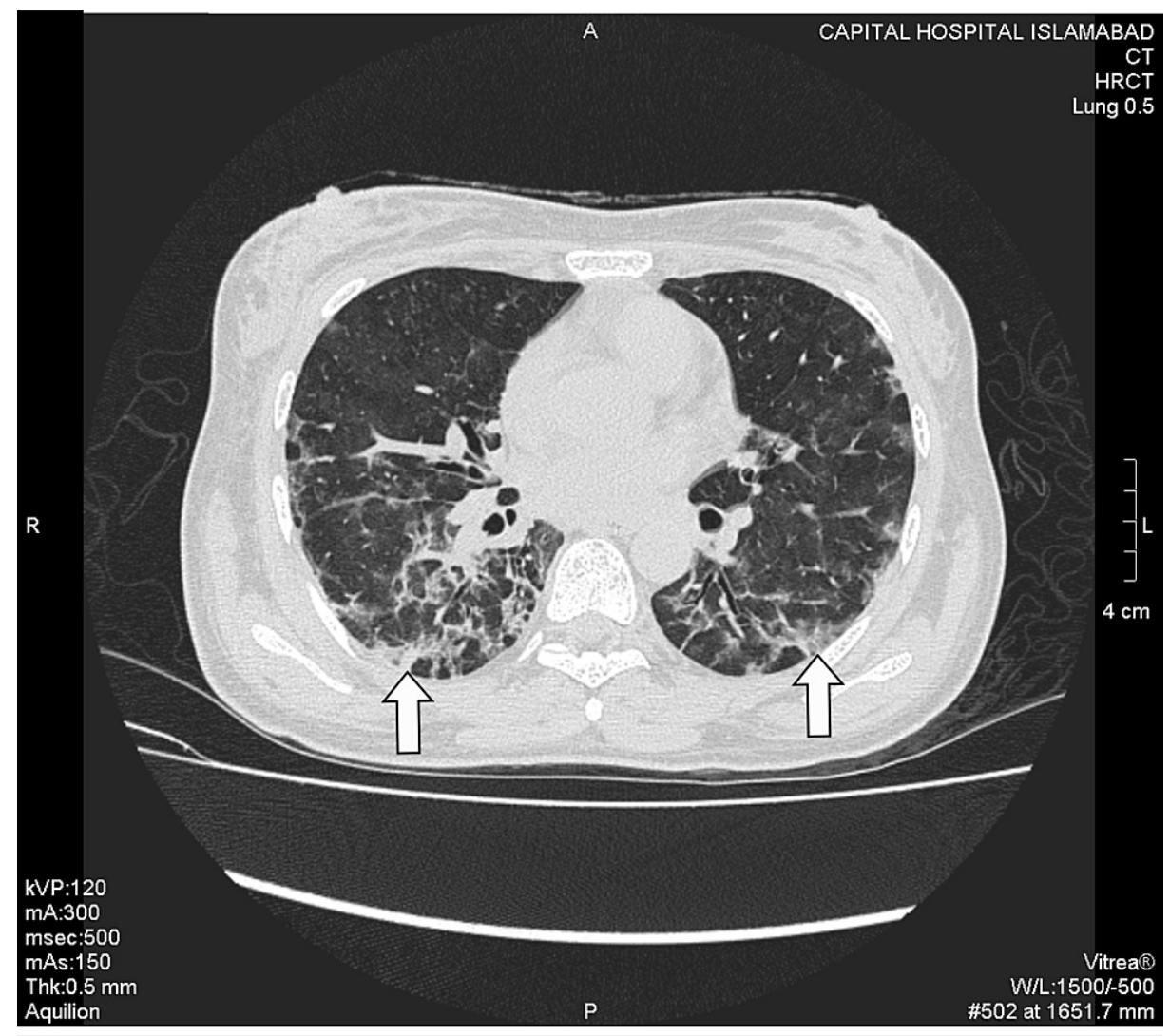

FIGURE 5: A female patient with severe symptoms.

HRCT shows multiple ground-glass opacities mixed with consolidation in bilateral lower lobes (white arrows). HRCT: High-resolution computed tomography. 


\section{Cureus}

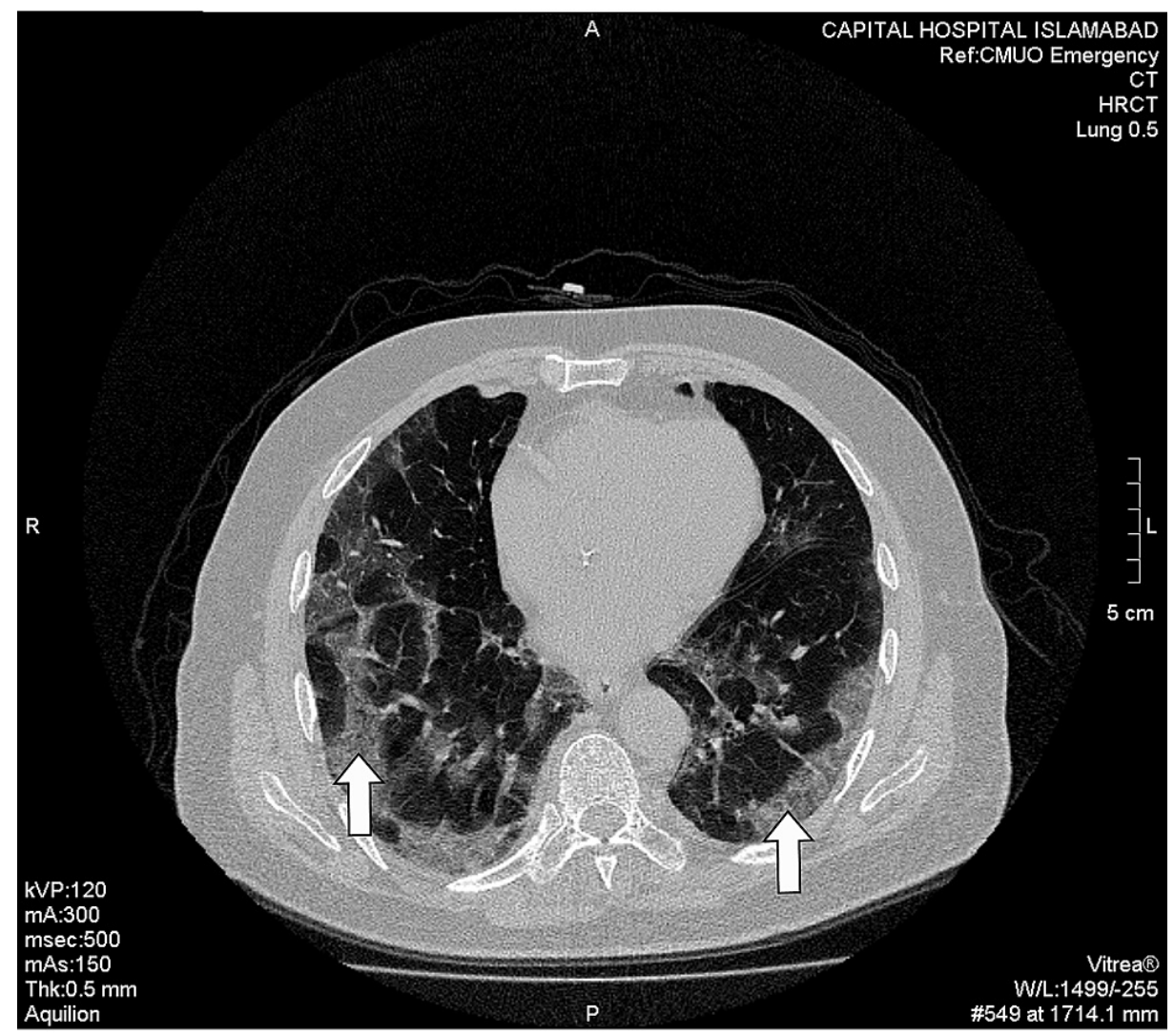

FIGURE 6: A 78-year-old male patient with shortness of breath and oxygen saturation less than $85 \%$ and dropping.

HRCT scan exhibits severe disease with multiple predominantly peripheral-based large patches of groundglass opacities along with a crazy-paving pattern scattered in bilateral lung fields (white arrows).

HRCT: High-resolution computed tomography.

\begin{tabular}{|c|c|c|c|}
\hline S. No & Radiological findings & Frequency & Percentage (Out of 38) \\
\hline 1 & Ground-glass opacities & 7 & $18.42 \%$ \\
\hline 2 & Ground-glass opacities with a crazy-paving pattern & 22 & $57.89 \%$ \\
\hline 3 & Mixed ground-glass opacities and consolidation & 9 & $23.68 \%$ \\
\hline 4 & Subpleural atelectatic bands & 18 & $47.36 \%$ \\
\hline 5 & Prominent bronchovascular markings & 15 & $39.47 \%$ \\
\hline 6 & Reverse halo sign & 9 & $23.68 \%$ \\
\hline
\end{tabular}

\section{TABLE 2: HRCT chest features suggestive of COVID-19.}

HRCT: High-resolution computed tomography.

Among 38 HRCT scans, 97.37\% had evidence of multifocal lesions in contrast to the unifocal lesions (Figures $2,3,4,5,6$ ). Lobar distribution of opacities in lung parenchyma was as follows: right upper lobe $42.10 \%$, right middle lobe $55.2 \%$, right lower lobe $81.6 \%$, left upper lobe $10.5 \%$, and left lower lobe $76.3 \% .92 .10 \%$ of these scans had bilateral and 7.89\% had unilateral lesions (Figures 2, 3, 4, 5, 6). In 94.74\% cases, the lesions were in peripheral, $5.26 \%$ central, $84.21 \%$ dorsal, and $15.79 \%$ in ventral distribution respectively (Figures 2 , $3,4,5,6)$. 


\section{Discussion}

Coronavirus disease is a rapidly spreading infection being characterized as a global pandemic [2]. The HRCT chest and RT-PCR assay are both unable to detect COVID-19 infection in the early stages. However, HRCT chest is a rapid method of identifying COVID-19 infected patients compared to RT-PCR assay which requires at least a day or two for the results, and thus, it can play a significant role in the control of the spread of infection especially in the emergency state of the rapid spread of COVID-19 [6]. The inconsistent and low sensitivity of the RT-PCR test indicates that numerous COVID-19 patients cannot be diagnosed leading to a rapid spread in the community [9]. CT chest has been adopted in the diagnostic criteria of COVID-19 for some time being in Hubei, China [13].

Our study determined non-contrast HRCT chest findings in symptomatic COVID-19 cases with an initial negative RT-PCR assay. The time interval between RT-PCR assay and pulmonary HRCT was kept at 0-7 days in the study by Ai et al whereas in our study the time interval was restricted from 0-3 days with a median of one day [14].

According to studies by Xu et al and Ai et al, 31.8\% and 75\% respectively of RT-PCR negative highly suspected cases had positive findings on HRCT scans characteristically of COVID-19. Our study had similar results to the study done by Ai et al with $79.2 \%$ of suspected cases showing HRCT features suggestive of the infection $[13,14]$.

A study done in London showed $20 \%$ of lesions to be pure ground-glass opacities and $80 \%$ of lesions to be mixed GGO and consolidation whereas our study indicated that $18.42 \%$ of ground-glass opacities were pure and only $23.68 \%$ were mixed with patches of consolidation. This study showed crazy paving in no patients with subpleural fibrotic lines in $60 \%$ of the cases compared to our study which showed crazy paving in $57.89 \%$ and subpleural atelectatic bands in $47.36 \%$ of the cases. The same study demonstrated the distribution of ground-glass opacities to be bilateral in $100 \%$, peripheral and dorsal in $80 \%$ of the cases. In comparison, our study indicated $92.10 \%$ bilateral, $94.74 \%$ peripheral, and $84.21 \%$ dorsal ground-glass opacities. Lower lobes were involved in $100 \%$ of the cases with ground glass opacities in this study, on the other hand, our research demonstrated that $81.6 \%$ of patients with positive HRCT features had involvement of lower lobes. The same study manifested the ground glass opacities to be predominantly multifocal in nature. Similar to this, our study had $97.37 \%$ cases with multifocal lesions [15].

Non-contrast HRCT chest is superior to many other investigations being non-invasive to the patient. However, there are a few limitations to this procedure like its expense and high radiation exposure. The patient has to be shifted to CT scanners to perform an HRCT scan which is difficult for severely ill patients. GGO, the hallmark of COVID 19 on HRCT chest has a wide range of differential diagnoses and the diagnosis of the infection based on HRCT scan findings is subjective depending on the expertise of the radiologists varying from person to person.

However, this research showed that a significant number of COVID-19 pneumonia suspects with negative RT-PCR test had positive chest HRCT features consistent with the diagnosis of coronavirus disease which might be true positive owing to the less sensitive nature of RT-PCR assay. We recommend the use of an HRCT scan along with detailed history and clinical evaluation in patients with diagnostic uncertainty yet high clinical suspicion for COVID-19 infection. We also suggest future studies to be conducted to establish the accuracy of HRCT chest and its inclusion in the diagnostic regimen of patients with a high index of suspicion for coronavirus infection.

\section{Conclusions}

In the light of our conducted research, we can ascertain that the HRCT chest has paramount importance in suspected COVID-19 cases with diagnostic uncertainty. An initial negative RT-PCR test does not rule out the disease, particularly in highly suspicious patients. The only solution to manage the rapid spread of coronavirus disease is early identification and quarantine of COVID-19 patients, hence clinically doubtful COVID-19 cases showing positive features of the infection on the HRCT chest should be treated and isolated as positive cases despite being PCR negative.

\section{Additional Information \\ Disclosures}

Human subjects: Consent was obtained or waived by all participants in this study. Hospital Ethics Committee, Capital Hospital issued approval CDA/CH/ENT (Genl) (1) /2021/446. The committee authorized unconditional permission to proceed with this project. However, the committee reserved the right to stop the research project if reports were received regarding the causation of undue risks or hazards to the study of the patients. Animal subjects: All authors have confirmed that this study did not involve animal subjects or tissue. Conflicts of interest: In compliance with the ICMJE uniform disclosure form, all authors declare the following: Payment/services info: All authors have declared that no financial support was received from any organization for the submitted work. Financial relationships: All authors have declared that they have 
no financial relationships at present or within the previous three years with any organizations that might have an interest in the submitted work. Other relationships: All authors have declared that there are no other relationships or activities that could appear to have influenced the submitted work.

\section{References}

1. WHO Coronavirus (COVID-19) Dashboard. (2021). Accessed: February 02, 2021: https://covid19.who.int/..

2. Karia R, Gupta I, Khandait H, Yadav A, Yadav A: COVID-19 and its modes of transmission. SN Compr Clin Med. 2020, 2:1798-1801. 10.1007/s42399-020-00498-4

3. Atif M, Malik I: Why is Pakistan vulnerable to COVID-19 associated morbidity and mortality? A scoping review. Int J Health Plann Manage. 2020, 35:1041-1054. 10.1002/hpm.3016

4. Esakandari H, Nabi-Afjadi M, Fakkari-Afjadi J, Farahmandian N, Miresmaeili S-M, Bahreini E: A comprehensive review of COVID-19 characteristics. Biol Proced Online. 2020, 22:19. 10.1186/s12575-02000128-2

5. Baloch S, Baloch MA, Zheng T, Pei X: The coronavirus disease 2019 (COVID-19) pandemic . Tohoku J Exp Med. 2020, 250:271-278. 10.1620/tjem.250.271

6. Mahalmani VM, Mahendru D, Semwal A, et al.: COVID-19 pandemic: a review based on current evidence . Indian J Pharmacol. 2020, 52:117-129. 10.4103/ijp.IJP 31020

7. Koo HJ, Choi S-H, Sung H, Choe J, Do K-H: RadioGraphics update: radiographic and CT features of viral pneumonia. Radiographics. 2020, 40:E8-E15. 10.1148/rg.2020200097

8. Khaliq M, Raja R, Khan N, Hanif H: An analysis of high-resolution computed tomography chest manifestations of COVID-19 patients in Pakistan. Cureus. 2020, 12:e9373. 10.7759/cureus.9373

9. Young D, Tatarian L, Mujtaba G, et al.: Chest CT versus RT-PCR for diagnostic accuracy of COVID-19 detection: a meta-analysis. J Vasc Med Surg. 2020, 8:392. 10.35248/2329-6925.20.8.392

10. Gao L, Zhang J: Pulmonary high-resolution computed tomography (HRCT). Findings of patients with earlystage coronavirus disease 2019 (COVID-19) in Hangzhou, China. Med Sci Monit. 2020, 26:e923885-4. 10.12659/MSM.923885

11. Pascarella G, Strumia A, Piliego C, et al.: COVID-19 diagnosis and management: a comprehensive review . I Intern Med. 2020, 288:192-206. 10.1111/joim.13091

12. Gietema HA, Zelis N, Nobel JM, et al.: CT in relation to RT-PCR in diagnosing COVID-19 in The Netherlands: a prospective study. PLoS One. 2020, 15 :e0235844. 10.1371/journal.pone.0235844

13. Xu B, Xing Y, Peng J, et al.: Chest CT for detecting COVID- 19: a systematic review and meta-analysis of diagnostic accuracy. Eur Radiol. 2020, 30:5720-5727. 10.1007/s00330-020-06934-2

14. Ai T, Yang Z, Hou H, et al.: Correlation of chest CT and RT-PCR testing for coronavirus disease 2019 (COVID-19) in China: a report of 1014 cases. Radiology. 2020, 296:E32-E40. 10.1148/radiol.2020200642

15. Pakdemirli E, Mandalia U, Monib S: Positive chest CT features in patients with COVID-19 pneumonia and negative real-time polymerase chain reaction test. Cureus. 2020, 12:e9942. 10.7759/cureus.9942 